\title{
Interval-valued intuitionistic fuzzy graphs
}

\author{
Krassimir Atanassov ${ }^{1,2}$ \\ ${ }^{1}$ Bioinformatics and Mathematical Modelling Department \\ Institute of Biophysics and Biomedical Engineering, Bulgarian Academy of Sciences \\ 105 Acad. G. Bonchev Str., Sofia 1113, Bulgaria \\ ${ }^{2}$ Intelligent Systems Laboratory, Prof. Asen Zlatarov University \\ 1 Prof. Yakimov Blvd., Burgas 8010, Bulgaria \\ e-mail: krat@bas.bg
}

Received: 16 June 2018

Accepted: 24 January 2019

\begin{abstract}
The definitions of eight different types of interval-valued intuitionistic fuzzy graphs are introduced and their representations by index matrices are discussed. Examples for operations over these graphs are given.
\end{abstract}

Keywords: Interval-valued intuitionistic fuzzy graph, Interval-valued intuitionistic fuzzy set. 2010 Mathematics Subject Classification: 03E72, 05C10.

\section{Introduction}

The concept of the Intuitionistic Fuzzy Graph (IFG) was introduced by Anthony Shannon and the author in 1994 in [5]. In recent years, the concept was essentially extended and it found different applications.

Initially, following [2] we introduce for the first time definitions of Cartesian products over Interval-Valued Intuitionistic Fuzzy Sets (IVIFSs), while in [2] similar definitions are given only for the intuitionistic fuzzy sets (IFS) case.

Using these definitions, four definitions of Interval-Valued Intuitionistic Fuzzy Graphs (IVIFGs) will be given. After this, four more general definitions of IVIFGs will be introduced and some conditions for the correctness of these graphs will be discussed.

All definitions for IVIFSs are given in [2]. 


\section{Cartesian products over intuitionistic fuzzy sets and interval-valued intuitionistic fuzzy sets}

Let $E_{1}$ and $E_{2}$ be two universes and let

$$
\begin{aligned}
& A=\left\{\left\langle x, \mu_{A}(x), \nu_{A}(x)\right\rangle \mid x \in E_{1}\right\} \\
& B=\left\{\left\langle x, \mu_{B}(y), \nu_{B}(y)\right\rangle \mid y \in E_{2}\right\}
\end{aligned}
$$

be two IFSs; $A$ - over $E_{1}$ and $B$ - over $E_{2}$.

Following [2], we define:

$$
\begin{aligned}
A \times_{1} B= & \left\{\left\langle\langle x, y\rangle, \mu_{A}(x) \cdot \mu_{B}(y), \nu_{A}(x) \cdot \nu_{B}(y)\right\rangle \mid x \in E_{1} \& y \in E_{2}\right\}, \\
A \times_{2} B= & \left\{\left\langle\langle x, y\rangle, \mu_{A}(x)+\mu_{B}(y)-\mu_{A}(x) \cdot \mu_{B}(y), \nu_{A}(x) \cdot \nu_{B}(y)\right\rangle\right. \\
& \left.\mid x \in E_{1} \& y \in E_{2}\right\} \\
A \times_{3} B= & \left\{\left\langle\langle x, y\rangle, \mu_{A}(x) \cdot \mu_{B}(y), \nu_{A}(x)+\nu_{B}(y)-\nu_{A}(x) \cdot \nu_{B}(y)\right\rangle\right. \\
& \left.\mid x \in E_{1} \& y \in E_{2}\right\} \\
A \times_{4} B= & \left\{\left\langle\langle x, y\rangle, \min \left(\mu_{A}(x), \mu_{B}(y)\right), \max \left(\nu_{A}(x) \cdot \nu_{B}(y)\right)\right\rangle \mid x \in E_{1} \& y \in E_{2}\right\} \\
A \times_{5} B= & \left\{\left\langle\langle x, y\rangle, \max \left(\mu_{A}(x), \mu_{B}(y)\right), \min \left(\nu_{A}(x) \cdot \nu_{B}(y)\right)\right\rangle \mid x \in E_{1} \& y \in E_{2}\right\} .
\end{aligned}
$$

For the above two universes, let

$$
\begin{aligned}
& A=\left\{\left\langle x, M_{A}(x), N_{A}(x)\right\rangle \mid x \in E_{1}\right\}, \\
& B=\left\{\left\langle y, M_{B}(y), N_{B}(y)\right\rangle \mid y \in E_{2}\right\}
\end{aligned}
$$

be two IVIFSs; $A$ - over $E_{1}$ and $B$ - over $E_{2}$. Let each one of the intervals $M_{A}(x), N_{A}(x)$, $M_{B}(y), N_{B}(y)$ have the form $X=[\inf A, \sup X]$. Following the definition of an IVIFSs, we suppose that for each $x \in E_{1}, y \in E_{2}$ :

$$
M_{A}(x), N_{A}(x), M_{B}(y), N_{B}(y) \subseteq[0,1]
$$

and

$$
\sup M_{A}(x)+\sup N_{A}(x) \leq 1, \sup M_{B}(y)+\sup N_{B}(y) \leq 1 .
$$

Now, following [2], we define the following five Cartesian products over two IVIFSs and a new (sixth) one:

$$
\begin{aligned}
A \times_{1} B= & \left\{\left\langle\langle x, y\rangle,\left[\inf M_{A}(x) \inf M_{B}(y), \sup M_{A}(x) \sup M_{B}(y)\right],\right.\right. \\
& {\left.\left.\left[\inf N_{A}(x) \inf N_{B}(y), \sup N_{A}(x) \sup N_{B}(y)\right]\right\rangle \mid x \in E_{1} \& y \in E_{2}\right\}, } \\
A \times_{2} B= & \left\{\left\langle\langle x, y\rangle,\left[\inf M_{A}(x)+\inf M_{B}(y)-\inf M_{A}(x) \inf M_{B}(y),\right.\right.\right. \\
& \left.\sup M_{A}(x)+\sup M_{B}(y)-\sup M_{A}(x) \sup M_{B}(y)\right], \\
& {\left.\left.\left[\inf N_{A}(x) \inf N_{B}(y), \sup N_{A}(x) \sup N_{B}(y)\right]\right\rangle \mid x \in E_{1} \& y \in E_{2}\right\}, }
\end{aligned}
$$




$$
\begin{aligned}
A \times_{3} B= & \left\{\left\langle\langle x, y\rangle,\left[\inf M_{A}(x) \inf M_{B}(y), \sup M_{A}(x) \sup M_{B}(y)\right],\right.\right. \\
& {\left[\inf N_{A}(x)+\inf N_{B}(y)-\inf N_{A}(x) \inf N_{B}(y),\right.} \\
& \left.\left.\left.\sup N_{A}(x)+\sup N_{B}(y)-\sup N_{A}(x) \sup N_{B}(y)\right]\right\rangle \mid x \in E_{1} \& y \in E_{2}\right\}, \\
A \times_{4} B= & \left\{\left\langle\langle x, y\rangle,\left[\min \left(\inf M_{A}(x), \inf M_{B}(y)\right), \min \left(\sup M_{A}(x), \sup M_{B}(y)\right)\right],\right.\right. \\
& {\left.\left.\left[\max \left(\inf N_{A}(x) \inf N_{B}(y)\right), \max \left(\sup N_{A}(x) \sup N_{B}(y)\right)\right]\right\rangle \mid x \in E_{1} \& y \in E_{2}\right\} } \\
A \times_{5} B= & \left\{\left\langle\langle x, y\rangle,\left[\max \left(\inf M_{A}(x), \inf M_{B}(y)\right), \max \left(\sup M_{A}(x), \sup M_{B}(y)\right)\right],\right.\right. \\
& {\left.\left.\left[\min \left(\inf N_{A}(x), \inf N_{B}(y)\right), \min \left(\sup N_{A}(x), \sup N_{B}(y)\right)\right]\right\rangle \mid x \in E_{1} \& y \in E_{2}\right\}, } \\
A \times_{6} B= & \left\{\left\langle\langle x, y\rangle,\left[\frac{\inf M_{A}(x)+\inf M_{B}(y)}{2}, \frac{\sup M_{A}(x)+\sup M_{B}(y)}{2}\right],\right.\right. \\
& {\left.\left.\left[\frac{\inf N_{A}(x)+\inf N_{B}(y)}{2}, \frac{\sup N_{A}(x)+\sup N_{B}(y)}{2}\right]\right\rangle \mid x \in E_{1} \& y \in E_{2}\right\} . }
\end{aligned}
$$

Theorem. $A \times_{1} B, A \times_{2} B, A \times_{3} B, A \times_{4} B, A \times_{5} B, A \times_{6} B$ are IVIFSs.

Proof. We will prove that for every four real numbers $a, b, c, d \in[0,1]$, such that $a \leq c$, $b \leq d, c+d \leq 1$, the inequality

$$
c+d-c d-a-b+a b \geq 0
$$

holds. By a direct check we obtain that (1) is true for $a=c, b=d$, and for $a=0$ and $b=0$. In all other cases we have the equality $a b-c d=(a-c) b+(b-d) c$. Therefore, (1) is equivalent to:

$$
c-a+(a-c) b+d-b+(b-d) c=(c-a)(1-b)+(d-b)(1-c) \geq 0
$$

From the validity of (1) it follows that

$$
\begin{aligned}
& \sup M_{A}(x)+\sup M_{B}(y)-\sup M_{A}(x) \sup M_{B}(y), \\
& \geq \inf M_{A}(x)+\inf M_{B}(y)-\inf M_{A}(x) \inf M_{B}(y) .
\end{aligned}
$$

On the other hand, for every four real numbers $a, b, c, d \in[0,1]$, such that $a+b \leq 1, c+d \leq 1$, it is valid that

$$
a+c-a c+b d \leq a+c-a c+(1-a)(1-c)=1,
$$

i.e.,

$$
\sup M_{A}(x)+\sup M_{B}(y)-\sup M_{A}(x) \sup M_{B}(y)+\sup N_{A}(x) \sup N_{B}(y) \leq 1
$$

and hence $A \times_{2} B$ is an IVIFS. For the four other products the checks are analogous.

In [2], this Theorem has not been formulated and proved.

\section{First four types of interval-valued intuitionistic fuzzy graphs}

Let us have a (fixed) set of vertices $\mathcal{V}$. An (o)-IFG $G$ (over $\mathcal{V}$ ) will be the ordered pair $G=$ $\left(V^{*}, A^{*}\right)$, where 


$$
\begin{gathered}
V \subset \mathcal{V}, \\
V^{*}=\left\{\left\langle v, \mu_{V}(v), \nu_{V}(v)\right\rangle \mid v \in V\right\}, \\
A \subset V \times V, \\
A^{*}=\left\{\left\langle\langle x, y\rangle, \mu_{A}(x, y), \nu_{A}(x, y)\right\rangle \mid\langle x, y\rangle \in V \times V\right\}
\end{gathered}
$$

and functions $\mu_{V}: \mathcal{V} \rightarrow[0,1]$ and $\nu_{V}: \mathcal{V} \rightarrow[0,1]$ define the degree of membership and the degree of non-membership, respectively, of the element $v \in \mathcal{V}$ to the set $V$; the functions $\mu_{A}: E_{1} \times E_{2} \rightarrow[0,1]$ and $\nu_{A}: E_{1} \times E_{2} \rightarrow[0,1]$ define the degree of membership and the degree of non-membership, respectively, of the element $\langle x, y\rangle \in E_{1} \times E_{2}$ to the set $A \subseteq E_{1} \times E_{2}$; these functions have the forms of the corresponding components of the o-Cartesian product over IFSs, where $\circ \in\left\{\times_{1}, \times_{2}, \ldots, \times_{5}\right\}$ is an operation over IFSs, and for all $\langle x, y\rangle \in E_{1} \times E_{2}$,

$$
\begin{gathered}
0 \leq \mu_{V}(x)+\nu_{V}(x) \leq 1 \\
0 \leq \mu_{A}(x, y)+\nu_{A}(x, y) \leq 1 .
\end{gathered}
$$

The above definition is old (see, e.g., [2]), while the following three types of IVIFGs are introduced for the first time. Let us call the first definition (o)-(IFS, IFS)-IFG.

Now, we introduce the following three new definitions.

Let us have a (fixed) set of vertices $\mathcal{V}$. An (o)-(IFS, IVIFS)-IFG $G$ (over $\mathcal{V}$ ) will be the ordered pair $G=\left(V^{*}, A^{*}\right)$, where

$$
\begin{gathered}
V \subset \mathcal{V}, \\
V^{*}=\left\{\left\langle v, \mu_{V}(v), \nu_{V}(v)\right\rangle \mid v \in V\right\}, \\
A \subset V \times V, \\
A^{*}=\left\{\left\langle\langle x, y\rangle, M_{A}(x, y), N_{A}(x, y)\right\rangle \mid\langle x, y\rangle \in V \times V\right\}
\end{gathered}
$$

and functions $\mu_{V}: \mathcal{V} \rightarrow[0,1]$ and $\nu_{V}: \mathcal{V} \rightarrow[0,1]$ define the degree of membership and the degree of non-membership, respectively, of the element $v \in \mathcal{V}$ to the set $V$; the functions $M_{A}: E_{1} \times E_{2} \rightarrow \mathcal{P}([0,1])$ and $N_{A}: E_{1} \times E_{2} \rightarrow \mathcal{P}([0,1])$ define the degree of membership and the degree of non-membership, respectively, of the element $\langle x, y\rangle \in E_{1} \times E_{2}$ to the set $A \subseteq E_{1} \times E_{2}$, where for each set $Z, \mathcal{P}(Z)$ is the set of the subsets of $Z$; these functions have the forms of the corresponding components of the o-Cartesian product over IVIFSs, where $\circ \in\left\{\times_{1}, \times_{2}, \ldots, \times_{5}\right\}$ is an operation over IVIFSs, and for all $\langle x, y\rangle \in E_{1} \times E_{2}$,

$$
\begin{gathered}
0 \leq \mu_{V}(x)+\nu_{V}(x) \leq 1 \\
0 \leq \sup M_{A}(x)+\sup N_{A}(x) \leq 1 .
\end{gathered}
$$

Let us have a (fixed) set of vertices $\mathcal{V}$. An (o)-(IVIFS, IFS)-IFG $G$ (over $\mathcal{V}$ ) will be the ordered pair $G=\left(V^{*}, A^{*}\right)$, where 


$$
\begin{gathered}
V \subset \mathcal{V}, \\
V^{*}=\left\{\left\langle v, M_{V}(v), N_{V}(v)\right\rangle \mid v \in V\right\}, \\
A \subset V \times V, \\
A^{*}=\left\{\left\langle\langle x, y\rangle, \mu_{A}(x, y), \nu_{A}(x, y)\right\rangle \mid\langle x, y\rangle \in V \times V\right\}
\end{gathered}
$$

and functions $M_{V}: \mathcal{V} \rightarrow \mathcal{P}([0,1])$ and $N_{V}: \mathcal{V} \rightarrow \mathcal{P}([0,1])$ define the degree of membership and the degree of non-membership, respectively, of the element $v \in \mathcal{V}$ to the set $V$; functions $\mu_{A}: E_{1} \times E_{2} \rightarrow[0,1]$ and $\nu_{A}: E_{1} \times E_{2} \rightarrow[0,1]$ define the degree of membership and the degree of non-membership, respectively, of the element $\langle x, y\rangle \in E_{1} \times E_{2}$ to the set $A \subseteq E_{1} \times E_{2}$; these functions have the forms of the corresponding components of the $\circ$-Cartesian product over IFSs, where $\circ \in\left\{\times_{1}, \times_{2}, \ldots, \times_{5}\right\}$ is an operation over IFSs, and for all $\langle x, y\rangle \in E_{1} \times E_{2}$,

$$
\begin{gathered}
0 \leq \sup M_{V}(x)+\sup N_{V}(x) \leq 1, \\
0 \leq \mu_{A}(x, y)+\nu_{A}(x, y) \leq 1
\end{gathered}
$$

Let us have a (fixed) set of vertices $\mathcal{V}$. An (o)-(IVIFS, IVIFS)-IFG $G$ (over $\mathcal{V}$ ) will be the ordered pair $G=\left(V^{*}, A^{*}\right)$, where

$$
\begin{gathered}
V \subset \mathcal{V}, \\
V^{*}=\left\{\left\langle v, M_{V}(v), N_{V}(v)\right\rangle \mid v \in V\right\}, \\
A \subset V \times V, \\
A^{*}=\left\{\left\langle\langle x, y\rangle, M_{A}(x, y), N_{A}(x, y)\right\rangle \mid\langle x, y\rangle \in V \times V\right\}
\end{gathered}
$$

and functions $M_{V}: \mathcal{V} \rightarrow \mathcal{P}([0,1])$ and $N_{V}: \mathcal{V} \rightarrow \mathcal{P}([0,1])$ define the degree of membership and the degree of non-membership, respectively, of the element $v \in \mathcal{V}$ to the set $V$; the functions $M_{A}: E_{1} \times E_{2} \rightarrow \mathcal{P}([0,1])$ and $\nu_{A}: E_{1} \times E_{2} \rightarrow \mathcal{P}([0,1])$ define the degree of membership and the degree of non-membership, respectively, of the element $\langle x, y\rangle \in E_{1} \times E_{2}$ to the set $A \subseteq E_{1} \times E_{2}$; these functions have the forms of the corresponding components of the $\circ$-Cartesian product over IVIFSs, where $\circ \in\left\{\times_{1}, \times_{2}, \ldots, \times_{5}\right\}$ is an operation over IVIFSs, and for all $\langle x, y\rangle \in E_{1} \times E_{2}$ :

$$
\begin{gathered}
0 \leq \sup M_{V}(x)+\sup N_{V}(x) \leq 1, \\
0 \leq \sup M_{A}(x, y)+\sup N_{A}(x, y) \leq 1 .
\end{gathered}
$$

As in [4] and by analogy with [2], we illustrate the last of the above definitions by an example of a Berge's graph (see Fig. 1; the labels of the vertices and arcs show the corresponding degrees). Let the following Index Matrix (IM, see, e.g. [3]) giving $M$ - and $N$-values be defined for its $A$-values (for example, the data can be obtained as a result of some observations). 


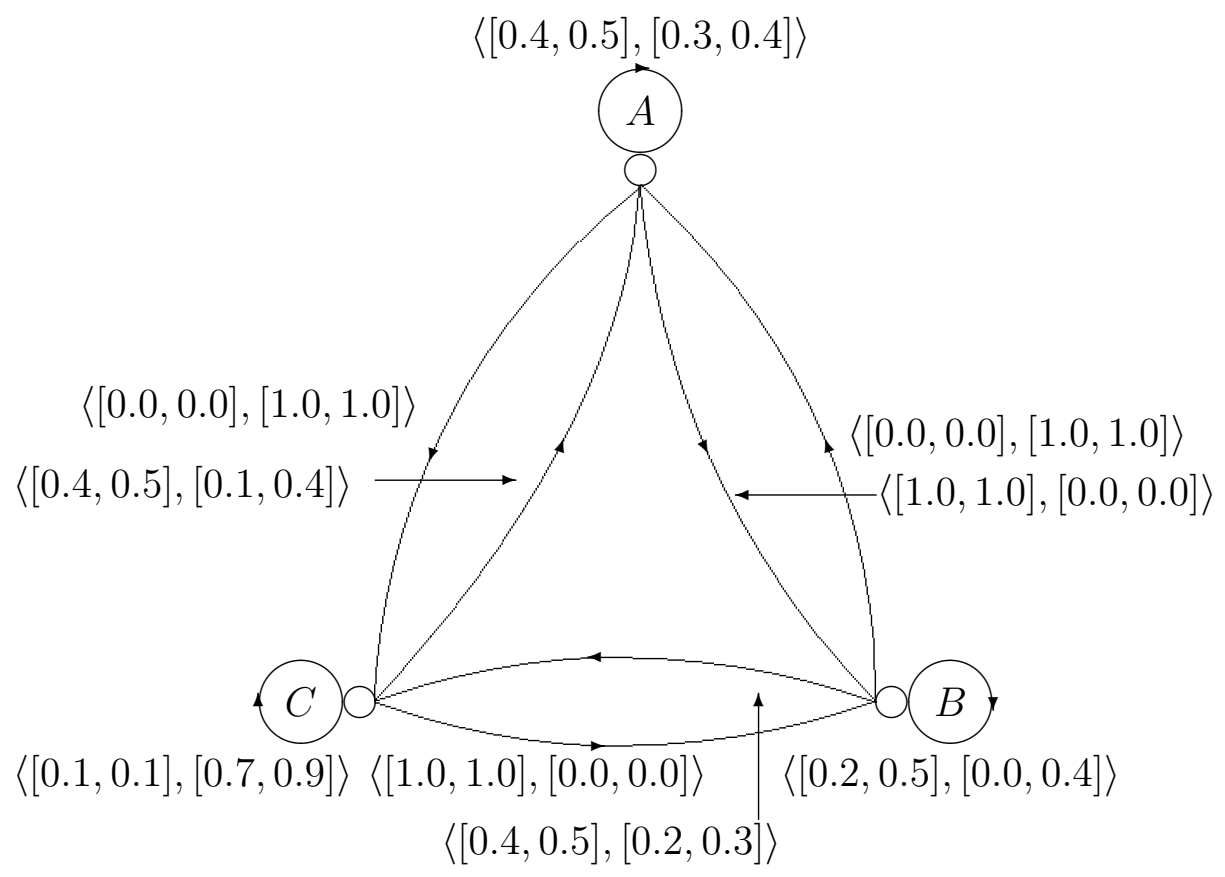

Figure 1.

\begin{tabular}{c|ccc}
$\left\langle M_{A}, N_{A}\right\rangle$ & $A$ & $B$ & $C$ \\
\hline$A$ & $\langle[0.4,0.5],[0.3,0.4]\rangle$ & $\langle[1.0,1.0],[0.0,0.0]\rangle$ & $\langle[0.0,0.0],[1.0,1.0]\rangle$ \\
$B$ & $\langle[0.0,0.0],[1.0,1.0]\rangle$ & $\langle[0.2,0.5],[0.0,0.4]\rangle$ & $\langle[0.4,0.5],[0.2,0.3]\rangle$ \\
$C$ & $\langle[0.4,0.5],[0.1,0.4]\rangle$ & $\langle[1.0,1.0],[0.0,0.0]\rangle$ & $\langle[0.1,0.1],[0.7,0.9]\rangle$
\end{tabular}

Having in mind that each real number $r$ can be represented as an interval $[r, r]$, we see that the first three types of graphs are partial cases of the fourth type.

\section{Second four types of interval-valued intuitionistic fuzzy graphs}

The new graphs have similar to the above form, but without the condition for the forms of their elements $\mu_{G}$ and $\nu_{G}$, or $M_{G}$ and $N_{G}$ elements. So, their definitions are the following.

Let us have a (fixed) set of vertices $\mathcal{V}$. An (IFS, IFS)-IFG $G$ (over $\mathcal{V}$ ) will be the ordered pair $G=\left(V^{*}, A^{*}\right)$, where

$$
\begin{gathered}
V \subset \mathcal{V}, \\
V^{*}=\left\{\left\langle v, \mu_{V}(v), \nu_{V}(v)\right\rangle \mid v \in V\right\}, \\
A \subset V \times V, \\
A^{*}=\left\{\left\langle\langle x, y\rangle, \mu_{A}(x, y), \nu_{A}(x, y)\right\rangle \mid\langle x, y\rangle \in V \times V\right\}
\end{gathered}
$$


and functions $\mu_{V}: \mathcal{V} \rightarrow[0,1]$ and $\nu_{V}: \mathcal{V} \rightarrow[0,1]$ define the degree of membership and the degree of non-membership, respectively, of the element $v \in \mathcal{V}$ to the set $V$; functions $\mu_{A}: E_{1} \times E_{2} \rightarrow[0,1]$ and $\nu_{A}: E_{1} \times E_{2} \rightarrow[0,1]$ define the degree of membership and the degree of non-membership, respectively, of the element $\langle x, y\rangle \in E_{1} \times E_{2}$ to the set $A \subseteq E_{1} \times E_{2}$; and for all $\langle x, y\rangle \in E_{1} \times E_{2}$,

$$
\begin{gathered}
0 \leq \mu_{V}(x)+\nu_{V}(x) \leq 1, \\
0 \leq \mu_{A}(x, y)+\nu_{A}(x, y) \leq 1 .
\end{gathered}
$$

Let us have a (fixed) set of vertices $\mathcal{V}$. An (IFS, IVIFS)-IFG $G$ (over $\mathcal{V}$ ) will be the ordered pair $G=\left(V^{*}, A^{*}\right)$, where

$$
\begin{gathered}
V \subset \mathcal{V}, \\
V^{*}=\left\{\left\langle v, \mu_{V}(v), \nu_{V}(v)\right\rangle \mid v \in V\right\}, \\
A \subset V \times V, \\
A^{*}=\left\{\left\langle\langle x, y\rangle, M_{A}(x, y), N_{A}(x, y)\right\rangle \mid\langle x, y\rangle \in V \times V\right\}
\end{gathered}
$$

and functions $\mu_{V}: \mathcal{V} \rightarrow[0,1]$ and $\nu_{V}: \mathcal{V} \rightarrow[0,1]$ define the degree of membership and the degree of non-membership, respectively, of the element $v \in \mathcal{V}$ to the set $V$; functions $M_{A}: E_{1} \times E_{2} \rightarrow \mathcal{P}([0,1])$ and $\nu_{A}: E_{1} \times E_{2} \rightarrow \mathcal{P}([0,1])$ define the degree of membership and the degree of non-membership, respectively, of the element $\langle x, y\rangle \in E_{1} \times E_{2}$ to the set $A \subseteq E_{1} \times E_{2} ;$ and for all $\langle x, y\rangle \in E_{1} \times E_{2}$,

$$
\begin{gathered}
0 \leq \mu_{V}(x)+\nu_{V}(x) \leq 1, \\
0 \leq \sup M_{A}(x)+\sup N_{A}(x) \leq 1 .
\end{gathered}
$$

Let us have a (fixed) set of vertices $\mathcal{V}$. An (IVIFS, IFS)-IFG $G$ (over $\mathcal{V}$ ) will be the ordered pair $G=\left(V^{*}, A^{*}\right)$, where

$$
\begin{gathered}
V \subset \mathcal{V}, \\
V^{*}=\left\{\left\langle v, M_{V}(v), N_{V}(v)\right\rangle \mid v \in V\right\}, \\
A \subset V \times V, \\
A^{*}=\left\{\left\langle\langle x, y\rangle, \mu_{A}(x, y), \nu_{A}(x, y)\right\rangle \mid\langle x, y\rangle \in V \times V\right\}
\end{gathered}
$$

and functions $M_{V}: \mathcal{V} \rightarrow \mathcal{P}([0,1])$ and $N_{V}: \mathcal{V} \rightarrow \mathcal{P}([0,1])$ define the degree of membership and the degree of non-membership, respectively, of the element $v \in \mathcal{V}$ to the set $V$; functions $\mu_{A}: E_{1} \times E_{2} \rightarrow[0,1]$ and $\nu_{A}: E_{1} \times E_{2} \rightarrow[0,1]$ define the degree of membership and the degree of non-membership, respectively, of the element $\langle x, y\rangle \in E_{1} \times E_{2}$ to the set $A \subseteq E_{1} \times E_{2}$; and for all $\langle x, y\rangle \in E_{1} \times E_{2}$,

$$
\begin{gathered}
0 \leq \sup M_{V}(x)+\sup N_{V}(x) \leq 1, \\
0 \leq \mu_{A}(x, y)+\nu_{A}(x, y) \leq 1 .
\end{gathered}
$$

Let us have a (fixed) set of vertices $\mathcal{V}$. An (IVIFS, IVIFS)-IFG $G$ (over $\mathcal{V}$ ) will be the ordered pair $G=\left(V^{*}, A^{*}\right)$, where 


$$
\begin{gathered}
V \subset \mathcal{V}, \\
V^{*}=\left\{\left\langle v, M_{V}(v), N_{V}(v)\right\rangle \mid v \in V\right\}, \\
A \subset V \times V, \\
A^{*}=\left\{\left\langle\langle x, y\rangle, M_{A}(x, y), N_{A}(x, y)\right\rangle \mid\langle x, y\rangle \in V \times V\right\}
\end{gathered}
$$

and functions $M_{V}: \mathcal{V} \rightarrow \mathcal{P}([0,1])$ and $N_{V}: \mathcal{V} \rightarrow \mathcal{P}([0,1])$ define the degree of membership and the degree of non-membership, respectively, of the element $v \in \mathcal{V}$ to the set $V$; functions $M_{A}: E_{1} \times E_{2} \rightarrow \mathcal{P}([0,1])$ and $\nu_{A}: E_{1} \times E_{2} \rightarrow \mathcal{P}([0,1])$ define the degree of membership and the degree of non-membership, respectively, of the element $\langle x, y\rangle \in E_{1} \times E_{2}$ to the set $A \subseteq E_{1} \times E_{2} ;$ and for all $\langle x, y\rangle \in E_{1} \times E_{2}$,

$$
\begin{gathered}
0 \leq \sup M_{V}(x)+\sup N_{V}(x) \leq 1 \\
0 \leq \sup M_{A}(x, y)+\sup N_{A}(x, y) \leq 1
\end{gathered}
$$

Obviously, the first four definitions are partial cases of the new four definitions, respectively.

\section{Remarks on the eight types of interval-valued intuitionistic fuzzy graphs}

From [3], it is clear that in the general case, if $V=\left\{v_{1}, v_{2}, \ldots, v_{n}\right\}$, then the index matrix (IM) of the first, second, fifth and sixth graphs can have the form

$$
A=\begin{array}{c|cccc} 
& v_{1} & v_{2} & \cdots & v_{n} \\
\hline v_{1} & a_{1,1} & a_{1,2} & \cdots & a_{1, n} \\
v_{2} & a_{2,1} & a_{2,2} & \cdots & a_{2, n} \\
\vdots & \vdots & \vdots & \ddots & \vdots \\
v_{n} & a_{n, 1} & a_{n, 2} & \cdots & a_{n, n}
\end{array}
$$

where $n$ is the cardinality of set $V$ and

$$
\begin{aligned}
a_{i, j}=\left\langle\mu_{i, j}, \nu_{i, j}\right\rangle & \in[0,1] \times[0,1](1 \leq i, j \leq n), \\
0 & \leq \mu_{i, j}, \nu_{i, j} \leq 1
\end{aligned}
$$

while the third, fourth, seventh and eighth graphs can have the form of the same IM, but now

$$
\begin{gathered}
a_{i, j}=\left\langle M_{i, j}, N_{i, j}\right\rangle \subseteq[0,1] \times[0,1](1 \leq i, j \leq n), \\
0 \leq \sup M_{i, j}+\sup N_{i, j} \leq 1 .
\end{gathered}
$$

Now, we can represent each of the four types of graphs in IM-form as

$$
G=\left[V^{*}, V^{*},\left\{a_{i, j}\right\}\right]
$$


Following the ideas from [3], it can be easily seen that the above IM can be modified to the following form:

$$
G=\left[V_{I}^{*} \cup \bar{V}^{*}, \bar{V}^{*} \cup V_{O}^{*},\left\{a_{i, j}\right\}\right],
$$

where $V_{I}^{*}, V_{O}^{*}$ and $\bar{V}^{*}$ are respectively the sets of the input, output and internal vertices of the graph. At least one arc leaves every vertex of the first type, but none enters; at least one arc enters each vertex of the second type but none leaves it; every vertex of the third type has at least one arc ending in it and at least one arc starting from it.

Obviously, the graph matrix (in the sense of IM) now will be of a smaller dimension than the ordinary graph matrix. Moreover, it can be non-square, unlike the ordinary graph, matrices.

As in the ordinary case, the vertex $v_{p} \in \bar{V}$ has a loop if and only if $a_{p, p}=\left\langle\mu_{p, p}, \nu_{p, p}\right\rangle$ for the vertex $v_{p}$ and $\mu_{p, p}>0$ (hence $\nu_{p, p}<1$ ).

Let us write below for brevity $G$ instead of $G^{*}$ and $V$ instead of $V^{*}$.

Let the graphs $G_{1}$ and $G_{2}$ be given and let

$$
G_{s}=\left[V_{s}^{\prime}, V_{s}^{\prime \prime},\left\{a_{i, j}^{s}\right\}\right]
$$

where $s=1,2$ and $V_{s}^{\prime}$ and $V_{s}^{\prime \prime}$ are the sets of the graph vertices (input and internal, and output and internal, respectively).

Then, using the apparatus of the IMs, we construct the graph which is a union of the graphs $G_{1}$ and $G_{2}$. The new graph has the description

$$
G=G_{1} \cup G_{2}=\left[V_{1}^{\prime} \cup V_{2}^{\prime}, V_{1}^{\prime \prime} \cup V_{2}^{\prime \prime},\left\{\bar{a}_{i, j}\right\}\right],
$$

where $\bar{a}_{i, j}$ is determined by the respective IM-formulas for operation $\cup$ from [3].

Analogously, we can construct a graph which is the intersection of the two given graphs $G_{1}$ and $G_{2}$. It would have the form

$$
G=G_{1} \cap G_{2}=\left[V_{1}^{\prime} \cap V_{2}^{\prime}, V_{1}^{\prime \prime} \cap V_{2}^{\prime \prime},\left\{\overline{\bar{a}}_{i, j}\right\}\right],
$$

where $\overline{\bar{a}}_{i, j}$ is determined by the respective IM-formulas for operation $\cap$ from [3].

\section{Level operators over interval-valued intuitionistic fuzzy sets and interval-valued intuitionistic fuzzy graphs}

These operators are introduced over IFSs in the first paper in this area - [1], and a part of them, over IVIFSs in [2]. First, we give the original definitions.

Let $\alpha, \beta \in[0,1]$ be fixed numbers for which $\alpha+\beta \leq 1$ and let

$$
\begin{aligned}
& N_{\alpha}^{1}(A)=\left\{\left\langle x, M_{A}(A), N_{A}(x)\right\rangle \mid x \in E \& \inf M_{A}(x) \geq \alpha\right\}, \\
& N_{1}^{\beta}(A)=\left\{\left\langle x, M_{A}(A), N_{A}(x)\right\rangle \mid x \in E \& \sup N_{A}(x) \leq \beta\right\}, \\
& N_{\alpha, \beta}^{1}(A)=\left\{\left\langle x, M_{A}(A), N_{A}(x)\right\rangle \mid x \in E \& \inf M_{A}(x) \geq \alpha \& \sup N_{A}(x) \leq \beta\right\},
\end{aligned}
$$




$$
\begin{aligned}
& N_{\alpha}^{2}(A)=\left\{\left\langle x, M_{A}(A), N_{A}(x)\right\rangle \mid x \in E \& \sup M_{A}(x) \geq \alpha\right\}, \\
& N_{2}^{\beta}(A)=\left\{\left\langle x, M_{A}(A), N_{A}(x)\right\rangle \mid x \in E \& \inf N_{A}(x) \leq \beta\right\}, \\
& N_{\alpha, \beta}^{2}(A)=\left\{\left\langle x, M_{A}(A), N_{A}(x)\right\rangle \mid x \in E \& \sup M_{A}(x) \geq \alpha \& \inf N_{A}(x) \leq \beta\right\}, \\
& N_{\alpha}^{3}(A)=\left\{\left\langle x, M_{A}(A), N_{A}(x)\right\rangle \mid x \in E \& \inf M_{A}(x) \leq \alpha\right\}, \\
& N_{3}^{\beta}(A)=\left\{\left\langle x, M_{A}(A), N_{A}(x)\right\rangle \mid x \in E \& \sup N_{A}(x) \geq \beta\right\}, \\
& N_{\alpha, \beta}^{3}(A)=\left\{\left\langle x, M_{A}(A), N_{A}(x)\right\rangle \mid x \in E \& \inf M_{A}(x) \leq \alpha \& \sup N_{A}(x) \geq \beta\right\}, \\
& N_{\alpha}^{4}(A)=\left\{\left\langle x, M_{A}(A), N_{A}(x)\right\rangle \mid x \in E \& \sup M_{A}(x) \leq \alpha\right\}, \\
& N_{4}^{\beta}(A)=\left\{\left\langle x, M_{A}(A), N_{A}(x)\right\rangle \mid x \in E \& \inf N_{A}(x) \geq \beta\right\}, \\
& N_{\alpha, \beta}^{4}(A)=\left\{\left\langle x, M_{A}(A), N_{A}(x)\right\rangle \mid x \in E \& \sup M_{A}(x) \leq \alpha \& \inf N_{A}(x) \geq \beta\right\},
\end{aligned}
$$

Now, we introduce four new operators

$$
\begin{aligned}
N_{\alpha, \beta, \gamma, \delta}^{5}(A)= & \left\{\left\langle x, M_{A}(A), N_{A}(x)\right\rangle \mid x \in E \& \inf M_{A}(x) \leq \alpha \& \sup M_{A}(x) \leq \beta\right. \\
& \left.\& \inf N_{A}(x) \geq \gamma \& \sup N_{A}(x) \geq \delta\right\}, \\
N_{\alpha, \beta, \gamma, \delta}^{6}(A)= & \left\{\left\langle x, M_{A}(A), N_{A}(x)\right\rangle \mid x \in E \& \inf M_{A}(x) \leq \alpha \& \sup M_{A}(x) \geq \beta\right. \\
& \left.\& \inf N_{A}(x) \geq \gamma \& \sup N_{A}(x) \leq \delta\right\}, \\
N_{\alpha, \beta, \gamma, \delta}^{7}(A)= & \left\{\left\langle x, M_{A}(A), N_{A}(x)\right\rangle \mid x \in E \& \inf M_{A}(x) \geq \alpha \& \sup M_{A}(x) \leq \beta\right. \\
& \left.\& \inf N_{A}(x) \leq \gamma \& \sup N_{A}(x) \geq \delta\right\}, \\
N_{\alpha, \beta, \gamma, \delta}^{8}(A)= & \left\{\left\langle x, M_{A}(A), N_{A}(x)\right\rangle \mid x \in E \& \inf M_{A}(x) \geq \alpha \& \sup M_{A}(x) \geq \beta\right. \\
& \left.\& \inf N_{A}(x) \leq \gamma \& \sup N_{A}(x) \leq \delta\right\} .
\end{aligned}
$$

We will call the above sets sets of $(\alpha, \beta)$-level generated by $A$.

Each of these operators can be applied over each of the eight types of interval-valued intuitionistic fuzzy graphs, but for this aim, we must modify them, because on the one hand they must be applied over $V$-elements, and on the other hand, must be applied over $A$-elements.

\section{Conclusion}

The introduced types of graphs will be object of further research. In near future, the author plans to study the possibility for applying different interval-valued intuitionistic fuzzy operators over these graphs. Also, different other types of graphs will be discussed (multigraphs, trees and others) and their applications in different areas will be searched, especially the areas of artificial intelligence, data mining and big data. 


\section{Acknowledgments}

The author is thankful for the support provided by the Bulgarian National Science Fund under Grant Ref. No. DN-02-10/2016 "New Instruments for Knowledge Discovery from Data, and their Modelling".

\section{References}

[1] Atanassov, K. (1983). Intuitionistic fuzzy sets, VII ITKR's Session, Sofia, June 1983 (Deposed in Central Sci. - Techn. Library of Bulg. Acad. of Sci., 1697/84, in Bulgarian). Reprinted: Int. J. Bioautomation, 2016, 20(S1), S1-S6.

[2] Atanassov, K. (1999). Intuitionistic Fuzzy Sets: Theory and Applications. Springer, Heidelberg.

[3] Atanassov, K. (2014). Index Matrices: Towards an Augmented Matrix Calculus, Springer, Cham.

[4] Kaufmann, A. (1977). Introduction a la Theorie des Sour-ensembles Flous, Paris, Masson.

[5] Shannon, A., \& Atanassov, K. (1994). A first step to a theory of the intuitionistic fuzzy graphs, Proc. of the First Workshop on Fuzzy Based Expert Systems (D. Lakov, Ed.), Sofia, Sept. 28-30, 1994, 59-61. 